\title{
Tombstone ST Segment Elevation in Double Vessel Coronary Artery Disease; "Who is the Culprit?"
}

Yutthapong Temtanakitpaisan*, Renee Dallasen and Craig S. Smith

Division of Cardiology, Department of Medicine, University of Massachusetts, Worcester, Massachusetts, USA

\begin{abstract}
We describe a 66 year-old male who presented with chest pain and ST segment elevation in the anterior leads. The typical culprit lesion is in the left anterior descending artery (LAD). However, concomitant lesions in the other coronary arteries may make an accurate diagnosis of the actual "culprit lesion" more challenging. Understanding the electrical basis of electrocardiograms can provide important clues in identifying and treating the true lesion.
\end{abstract}

\section{Introduction}

Acute ST segment elevation in the anterior chest leads typically represents acute infarction of the anterior wall of the left ventricle. The culprit lesion is usually found in the left anterior descending artery (LAD) or one of its branches. Isolated right ventricular infarction from right coronary artery (RCA) disease has been described as another cause of ST segment elevation in the anterior chest leads [1,2]. Identifying the actual "culprit lesion" becomes difficult when significant lesions are found concomitantly in the LAD and RCA.

\section{Case Presentation}

A 66 year-old male with a past medical history significant for hypertension and hyperlipidemia presented with acute substernal chest pain, which radiated to the left shoulder and was associated with nausea and diaphoresis. His initial electrocardiogram (ECG) demonstrated normal sinus rhythm with ST segment elevation in leads V2-V3 (Figure 1). Emergency cardiac catheterization was performed and revealed stenosis of the mid left anterior descending artery (LAD) at the origin of the first diagonal branch with Thrombolysis In Myocardial Infarction (TIMI) -3 flow with an angiographic radiolucent appearance consistent with thrombus (Figure 2). Intravascular ultrasound confirmed highgrade stenosis of the mid LAD lesion (Figure 3) with an eccentric, atheromatous core and minimal fibrous tissue. A second lesion was identified as a $100 \%$ stenosis of the acute marginal branch of the nondominant right coronary artery (RCA) (Figure 4a). The LAD lesion appeared to be the culprit lesion with spontaneous recanalization given the absence of regional wall motion abnormalities on left ventriculogram. Due to the small cross sectional diameter of the lesion, thrombectomy was not performed. The LAD lesion was predilated with a $3 \mathrm{~mm}$ balloon, and a XIENCE XP drug-eluting stent was deployed across the lesion with TIMI 3 resultant flow. After the intervention, the patient remained symptomatic with persistent ST segment elevation. Right sided chest leads were placed, which revealed $1 \mathrm{~mm} \mathrm{ST} \mathrm{segment}$ elevation in lead V4R (Figure 5). A stat transthoracic echocardiogram demonstrated normal left ventricular systolic function with no regional wall motion abnormalities with mild right ventricular dilation. The patient's first troponin I level was $9.51 \mathrm{ng} / \mathrm{ml}$ with a peak value of 15.02 $\mathrm{ng} / \mathrm{ml}$. Revascularization of the acute marginal branch of the RCA with balloon angioplasty was then performed, with restoration of TIMI-3 flow, after which a residual $50 \%$ stenosis in a small caliber vessel (2 $\mathrm{mm}$ ) was seen (Figure $4 \mathrm{~b}$ ). After the RV marginal intervention, the patient was chest pain free with improvement in ST segment elevation (Figure 6)

\section{Discussion}

ST segment elevation in leads V1-V3 is characteristic of LAD disease but has also been described in RCA occlusions [1,2]. When the RCA is dominant and gives rise to the posterior descending artery supplying the inferior wall and ventricular septum, occlusions proximal to the acute marginal branch cause both inferior wall and right ventricular infarcts. ECG typically demonstrates evidence of inferior injury, but electrocardiographic evidence of right ventricular infarction is suppressed by dominant electrical forces of simultaneous left ventricular inferoposterior injury [3,4]. In contrast, when a non-dominant RCA occlusion occurs in the absence of other native coronary artery disease, ST segment elevation in the anterior chest leads can be present due to absence of left ventricular injury altering electrical forces [2].

Our case demonstrates an isolated right ventricular injury from a lesion in the acute marginal branch of the RCA with no left

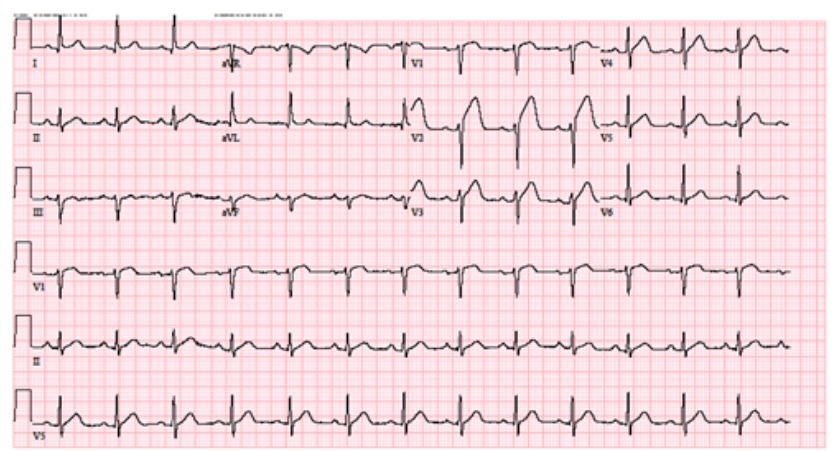

Figure 1: ECG demonstrates tombstone ST elevation in lead V2-V4.

*Corresponding author: Yutthapong Temtanakitpaisan, Division of Cardiology Department of Medicine, University of Massachusetts, 55 Lake Avenue North, Worcester, Massachusetts 01655, USA, Tel: 1-267-262-1720; E-mail: Yutthapong.temtanakitpaisan@umassmemorial.org

Received July 07, 2015; Accepted July 21, 2015; Published July 23, 2015

Citation: Temtanakitpaisan Y, Dallasen R, Smith CS (2015) Tombstone ST Segment Elevation in Double Vessel Coronary Artery Disease; "Who is the Culprit?". J Cardiovasc Dis Diagn 3: 211. doi:10.4172/2329-9517.1000211

Copyright: @ 2015 Temtanakitpaisan Y, et al. This is an open-access article distributed under the terms of the Creative Commons Attribution License, which permits unrestricted use, distribution, and reproduction in any medium, provided the original author and source are credited. 
Citation: Temtanakitpaisan Y, Dallasen R, Smith CS (2015) Tombstone ST Segment Elevation in Double Vessel Coronary Artery Disease; "Who is the Culprit?". J Cardiovasc Dis Diagn 3: 211. doi:10.4172/2329-9517.10002111000207

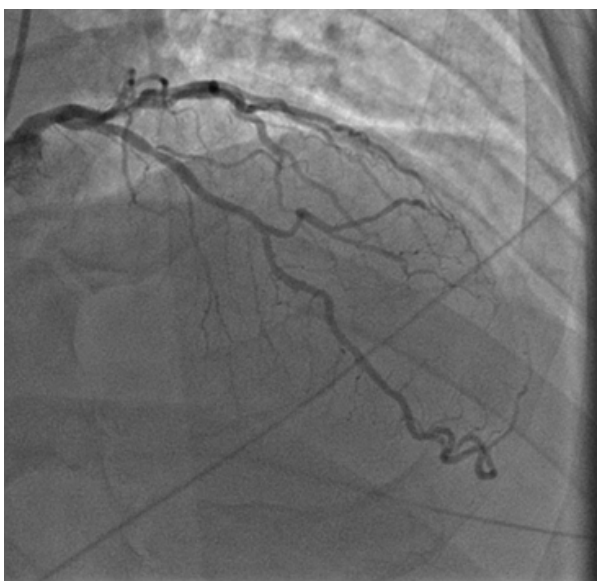

Figure 2: Left coronary angiogram shows $90 \%$ mid LAD disease.

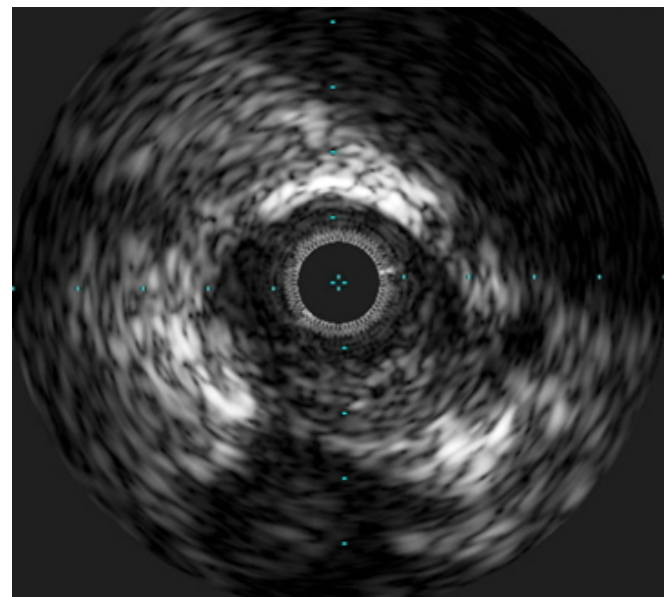

Figure 3: IVUS demonstrates significant LAD disease.

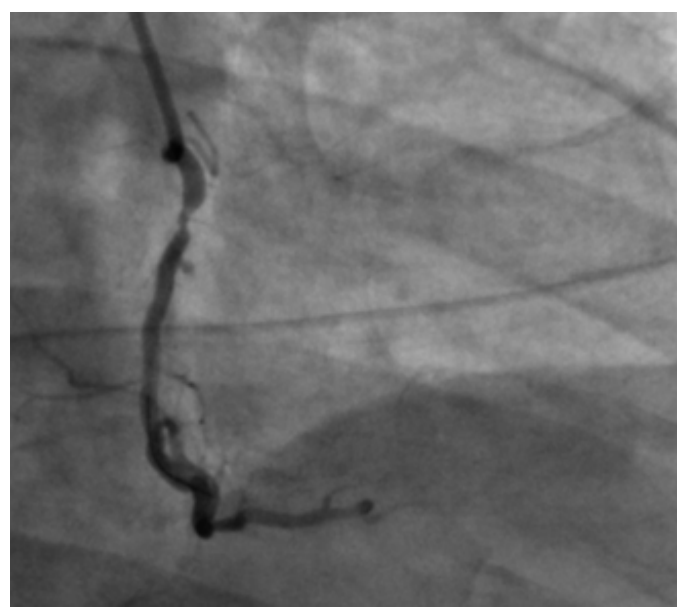

Figure 4a: Right coronary angiogram shows $100 \%$ stenosis of the acute marginal branch of RCA.

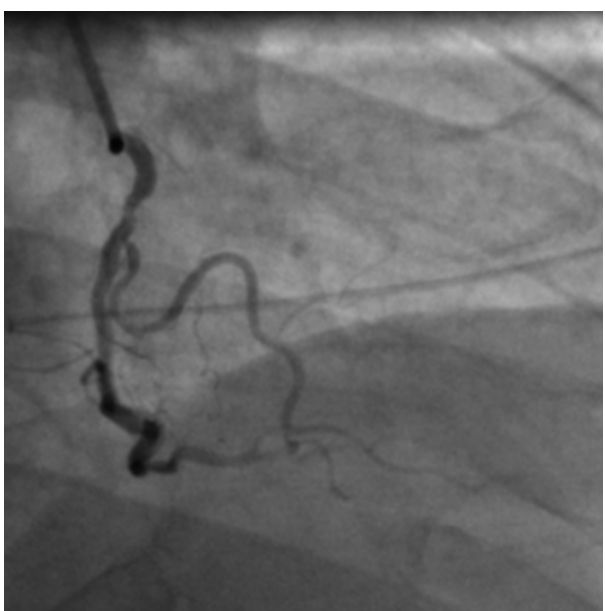

Figure 4b: Right coronary angiogram after revascularization of the acute marginal branch of the RCA shows restoration of TIMI-3 flow.

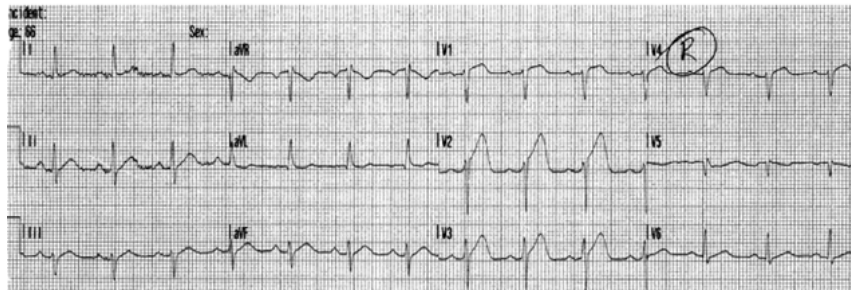

Figure 5: ECG reveals $1 \mathrm{~mm} \mathrm{ST} \mathrm{segment} \mathrm{elevation} \mathrm{in} \mathrm{lead} \mathrm{V4R}$

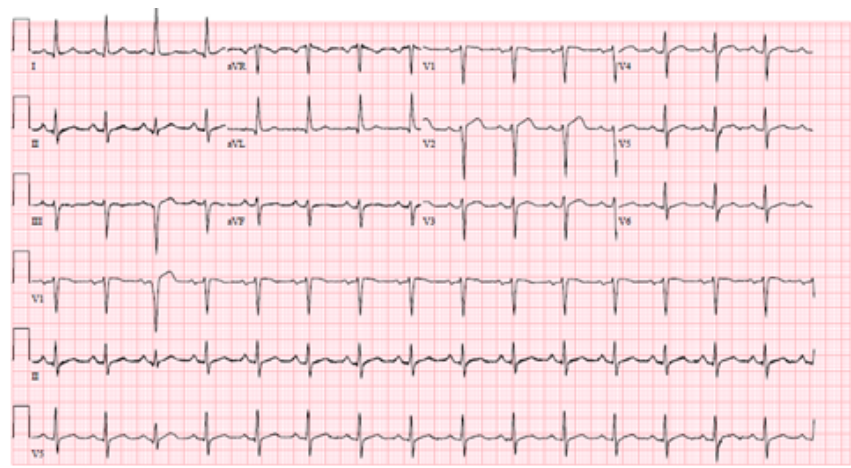

Figure 6: ECG after revascularization shows improvement of ST elevation in the anterior lead.

ventricular inferior wall involvement. As mentioned above, presence of simultaneous, angiographically significant lesions in the LAD and RCA can make it difficult to distinguish the culprit lesion. Moreover, IVUS confirmed a significant lesion in the LAD, which led us to blame an "innocent bystander" as the culprit lesion. When assessing tombstone ST segment elevation in the anterior leads, an isolated RCA occlusion should be considered when there is absence of left ventricular regional wall motion abnormalities or absence of reciprocal electrocardiographic changes in the inferior wall. 
Citation: Temtanakitpaisan Y, Dallasen R, Smith CS (2015) Tombstone ST Segment Elevation in Double Vessel Coronary Artery Disease; "Who is the Culprit?". J Cardiovasc Dis Diagn 3: 211. doi:10.4172/2329-9517.10002111000207

Page 3 of 3

\section{References}

1. Eichhofer J, Curzen N (2005) Images in cardiovascular medicine. Unexpected profound transient anterior ST elevation after occlusion of the conus branch of the right coronary artery during angioplasty. Circulation 111: e113-114.

2. Finn AV, Antman EM (2003) Images in clinical medicine. Isolated right ventricular infarction. N Eng J Med 349: 1636.

3. Geft IL, Shah PK, Rodriguez L, Hulse S, Maddahi J, et al. (1984) ST elevation in leads V1 to V 5 may be caused by right coronary artery occlusion and acute right ventricular infarction. Am J Cardiol 53: 991-996.

4. van der Bolt CL, Vermeersch PH, Plokker HW (1996) Isolated acute occlusion of a large right ventricular branch of the right coronary artery following coronary balloon angioplasty. The only true 'model' to study ECG changes in acute, isolated right ventricular infarction. Eur Heart J 17: 247-250. 